\title{
Pagtuklas sa mga piling maikling akda ni P. V. Villafuerte sa realismong pananaw
}

\author{
Mandado, Juliet O. $\triangle$ \\ Cebu Technological University - Main Campus, Cebu, Philippines (ctu2020school@gmail.com)
}

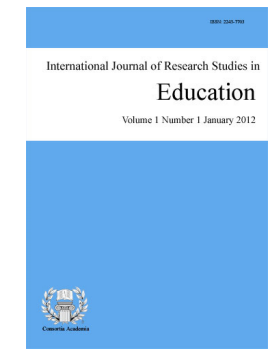

ISSN: $2243-7703$ Online ISSN: 2243-7711

OPEN ACCESS

\section{Abstract}

Filipinos are creative and expressive for the love of literature is natural. The purpose of this study is to present, analyze and recognize the importance of the short stories of Patrocinio V. Villafuerte who won the award from Carlos Palanca Memorial Awards for Literature in 1984 Ang Huling Itineraryo sa Pulu-pulong Utak ni Propesor Balisungsong, in 1991- Si Ato sa Sangmagdamagang Pagtakas sa Kawalang-Malay and another year in 2002 was Huling Hiling, Hinaing at Halinghing ni Hermano Huseng that all of Villafuerte's writings received praise. The study will ensure literary elements such as character, setting, plot, theme and emotion; presented social relations; and realism perspective on written works. This research is a descriptive study conducted to discover whether the winning stories possess the characteristics consistent with the theory of Scott (1974) and Salazar (1995). This is qualitative research using content analysis, examining the contents of the work of an award-winning writer in Filipino and Literature. It is learned from the study that the analysis of the works reflects the realistic discussion of human life with a complete change in a society that each story can be considered meaningful especially what the author conveys to anyone who reads it. This critique is important to cultivate and further develop Filipino literary works that will be especially helpful in learning and teaching in Filipino.

Keywords: author; story; writings; author elements; realism perspective 


\section{Pagtuklas sa mga piling maikling akda ni P. V. Villafuerte sa realismong pananaw}

\section{Introduksyon}

Ang mga Pilipino ay matulain, malikhain, mapagpahayag, mapanlikha at likas na mapagmahal sa panitikan. Sa panitikan ay binubuhay ng may akda ang kasaysayan sa pamamagitan ng paglimbag nito sa pasulat na anyo na nabibigyang buhay sa bawat pagbuklat ng mga pahina nito. Nasabi nina Kraenkel (2003) at Wallen (2003) na ang maikling kuwento ay makapagdulot ng mga pagbabago sa buhay. Ito ay kapupulutan ng aral mula sa mga mambabasa ng kuwento, nobela, tula at iba pa.

Lubhang masalimuot ang mga ito kapag nabasa ang mga akda, sadyang makikita ang mithiin ng isang manunulat na maihayag ito ng malikhain. Ang maikling katha ay isang salaysay ng isang mahalagang pangyayaring kinasasangkutan ng isa o ilang tauhan at may isang kakintalan o impresyon. Ito ay maaaring mauri batay sa sangkap na binibigyang-diin sa salaysay. Ang kritikal na realismo ay ang mga gawain ng isang lipunang burgis upang maipamalas ang mga aspetong may kapangitan at panlulupig nito sa pahayag ni Bernales et al. (2009).

Ang maikling kuwento ay akda ng mga manunulat na may samot-saring sining na pupukaw sa ating guni-guni, at damdamin na maaaninag ang pangarap o tiyak na tagumpay ng tao sa paghahagilap ng kaligayahan. Ang tatlong maikling katha ni Patrocinio Villasan Villafuerte ang kasali sa pag-aaral na nakatanggap ng Carlos Palanca Memorial Awards for Literature, nakabatay sa mga teorya nina Scott (1947) at Salazar (1995). Ayon kay Salazar (1995) lumilitaw din ang makatotohanang panitikan na nagpapakita ng tunay na mga pangyayari sa mga taong nagmulat sa siglong ito. Ang pahayag ni Scott (1947) ang pag-unwang panlipunan ay nakapagdaragdag ng lakas na mabahaging gingampanan sa pakikipag-ugnayan ng panlipunan.

Nasabi nina Kraenkel (2003) at Wallen (2003) na ang maikling kwento ay makapagdulot ng mga pagbabago sa buhay. Ang mga aral, mensahe at tema ang naging gabay sa mga gawing pag-araw-araw at baging bukas sa anomang hamon sa buhay. Sa mga akdang makatotohanang pahayag nakapokus ang isinulat ng isa sa mga tanyag na mananalaysay ng Pilipinas, si Patrocinio Villasan Villafuerte. May mga naisulat na akda, isang editor, kolumnista at retiradong Propesor. Nagwagi sa mga contest ng Carlos Palanca Memorial Awards for Literature, tumanggap ng Gawad Surian Gantimpalang Collantes sa Sanayasay. Kaya, naisipan ng mananaliksik na gawan ng pagsusuri nang hindi matatawarang galing ng pagsulat na aantig sa bawat damdamin ng mambabasa.

Mapagbiyang halaga ang mga maikling kuwento sa loob ng kurikulum para mapakilala ang mga dalubhasa sa mga sining ng pagsulat. Mabigyan ng pansin ang mga araling pampanitikan sa pagsusuring-basa sa mga akdang maka-Pilipino at mas mapahalagahan ang mga nilalaman. Ang pagtatalakay sa akdang pampanitikan ay mabubuhay lalo na sa lahat ng antas pang-edukasyon para hindi ito mawawala sa puso at isipan ng mga kabataan. Ganito ang pagpapakahulugan nina Bayang (2020) at Go-Aco (2020) sa kanilang ginawang pag-aaral na Mga obserbasyon ng Mga Kulturang Pambansang Pilipino mula sa Mga aralin sa panitikang asyano sa filipino ng K12 grade 9 module na ang maikling kuwento ay isang uri ng masining na pagsasalaysay. Ito ay naglalayong magbigay aral, mangaliw, at pumukaw ng damdamin. Nagpapahiwatig na mayaman ang akda sa panlipunang aspeto.

Sa ganitong paraan ay malaking tulong ito na mapatupad ang layunin ng Edukasyon na maitanim sa isipan ng mga kabataan ang kahalagahan ng panitikan sa kanilang pag-aaral. Maging mapagmatyag sa nangyayari sa lipunan. Ang sariling wika sa pamamagitan ng pagbasa at pagsulat ng Panitikang Filipino ay natutuhan kung ito ay pag-aralan ng ninuman. Isinaad ni Tablan (2011) sa kanyang artikulong "Kaisipang Sosyalismo sa mga Akda ni Amado V. Hernandez" na ang pagsulat ay dapat may layuning panlipunan. Sa halip na panaginip o pantasya, ang sino mang mangangatha ay dapat sumulat base sa kanyang pag-iisip at kamalayan na naka-angkla sa kanyang kapaligirang ginagalawan. Ang kamalayan ng manunulat o makata man ay sumasalamin sa kalagayan

16 Consortia Academia Publishing (A partner of Network of Professional Researchers and Educators) 
ng kanyang bansa at kapaligirang ginagalawan.

Ang pagganap sa lipunan, bilang guro sa Filipino ay mabigyang-halaga ang katha ng sinumang Pilipino na may malasakit sa sariling bayan. Inaasahan na magamit sa pagtuturo ang mga sinusuri at mapakinabangan ng mag-aaral sa mga kasanayang makukuha mula sa katha ng isang maka-Pilipinong manunulat. Isa na rin ang dahilan ay ang pagtugon sa batas R.A. 10533 na palawakin at gamitin ang mga lokal na gawa na naaayon sa kontekstong nagsasaad na magbubuo ng disenyo at mga detalye ng kurikulum sa pinabuting batayang edukasyon.

May kaugnayan ang pag-aaral na ito sa kasalukuyang pag-aaral dahil ito ay tumatalakay sa panunuring pampanitikan kung saan ang mga bagong teknolohiya ay hindi makakapalit sa yamang likha ng mga manunulat sa ginagampanan nitong pakikipag-ugnayan sa lipunan. Ang panitikan ang siyang lakas na nagpapakilos sa alinmang uri ng lipunan. Malaki ang naiambag ng panitkan tungo sa kultura upang sagayon ay mapabilis na mapaunlad at mabigyang-halaga ang sariling panitikan lalo na sa kalagayang lokal na konteksto dahil may mga kabataan na hindi sapat ang kaalaman sa pagbabalangkas ng mga akda kung ito ay gagamitin sa pagtuturo ng pagsusuri. Binanggit ni Dinglasan (2001) sa kanyang aklat "Kritisimong Pampanitikan" na ang kariktan ng isang akdang pampanitikan ay masusukat sa pamamagitan ng pagsusuri sa kabuuan at nilalaman ng mga ito. Sa ganitong paraan ang panunuri ay isang uri ng paglalahad na nag-aanalisa, kumikilatis, tumitimbang, at nag-uugnay ng isang paksa sa kasalukuyan.

Ang pag-aaral na ito ay nakatuon na mailahad, masuri at makilala ang kahalagahan ng maiikling kuwento ni Patrocinio V. Villafuerte na nagkamit ng gantimpala mula Carlos Palanca Memorial Awards for Literature ang tatlong akda na lahat nito ay nakatanggap ng papuri. Sa pag-aaral na ito ang mga elementong literari tulad ng tauhan, tagpuan, banghay, tema at damdamin; nailalahad na ugnayang panlipunan; at realismong pananaw sa mga akdang naisulat ay siyang bibigyang tuon. Hindi maiwasan na ang mag-aaral sa ngayon ay nakasandal kung ano ang nasa elektonikong gawa at iba pang pinagkukunan na walang katiyikan ang awtentindad.

Binalanggkas ni Abdilla sa kanyang aklat na "Mga Kuwentong ginto; Ang Maikling Kwentong Tagalog" ang maikling kuwento ay nagtataglay ng mga katangian: banghay, paningin, suliranin, paksang-diwa, himig, kakalasan, kasukdula, galaw. Ganito rin ang kay Dinglasan (2005) na ang maikling kuwento ay isang akdang pampanitikan na nasa anyong tuluyan at ito ay nabubuo sa pamamagitan ng mga pangungusap at talata na masilan ang pangungusap sa buhay at pangunahing tauhan.

Ang mga nabanggit na kaugnayan na literatura at pag-aaral ay malaking tulong para mapalawak ang kasalukuyan pag-aaral. Sinusuri sa pag-aaral na ito ang mga nilalaman ng akda nito. Alamin ang mga ugnayang panlipunan at litering gamit sa realismong pananaw. Bunga nito, nilalayon ng pag-aaral na matulungan ang mag-aaral at guro sa Filipino na maihatid ang impormasyon sa panunuring panitikan sa mga estudyante at guro sa Filipino.

\section{Resulta at pagtalakay}

Ang pagsusuri sa akdang pampanitikan ay isang gawaing pagtuklas sa mga nakatagong kaalaman sa bawat akdang isinulat ng manunulat. Ang mga importansyang dulot ng nagagawang pagtuklas ay ayon sa pagkakaiba ng pamantayan sa damdamin, panlasa, kaisipan sa mga taong hilig ang pagsusulat at pagbabasa ng mga kuwentong madaling matapos sa isang upuan lamang.

Ang bahaging ito ay nakatuon sa pagsusuri ng elementong ginamit ng manunulat sa kanyang katha. Dito mapapakita ang pormal na katangian, ayon kay Arrogante (1993) nagiging masining ang pagkatha sapagkat, nalalangkapan ng mga etilo at pagdulog, mga piling salita, buhay na parang nakikita at naririnig. Ang konseptong ginamit sa pagsusuri ay patotoo sa sinabi ni Villafuerte (2000) sa pag-aaral ni Cuizon (2014) na sa pagsusuri ng maiikling kwento dapat suriin ang elementong taglay nito: tauhan, tagpuan, banghay, tunggalian, simbolo, pahiwatig at pahayag. Makikita sa ibaba ang mga halimbawa ng elementong gamit ng bawat maikling 
Mandado, J. O.

katha. Ang mga salitang nasa kaliwang hanay ay elementong gamit ng bawat maikling katha ay ang tauhan, tagpuan, banghay, tema at damdaming pantaong nakapaloob sa mga katha ibang pangyayari. Sinasalamin ang mga akdang pampanitikan sa bawat yugto ng buhay at sa lipunan na pumapalibot sa tao. Kapupulutan ng aral na magsisilbing gabay sa magandang mithiin at magagamit rin sa gawaing pampagkatuto sa isang pagsusuri ng akda.

\section{Talahanayan 1}

Mga elementong gamit sa bawat akda

\begin{tabular}{|c|c|c|c|}
\hline & \multicolumn{3}{|c|}{ Mga Kuwento } \\
\hline & 1 & 2 & 3 \\
\hline Tauhan & $\begin{array}{l}\text { Hermano Huseng - umibig sa } \\
\text { kauri }\end{array}$ & Ato - inosenteng bata & $\begin{array}{l}\text { Propesor Balisungsong - Propesor ng } \\
\text { unibersidad }\end{array}$ \\
\hline Tagpuan & $\begin{array}{l}\text { Isang baryo na malayo sa } \\
\text { siyudad }\end{array}$ & Sa iskwater sa may Coastal Road & Iskwelahan \\
\hline Banghay & $\begin{array}{l}\text { Lihim na pag-ibig, pagyurak sa } \\
\text { dangal, pagtuklas }\end{array}$ & Pagtupad sa pangarap, pagkamusmos & $\begin{array}{l}\text { Maaepektuhan ang pasahod, } \\
\text { natatawang-naiinis }\end{array}$ \\
\hline Tema & $\begin{array}{l}\text { Bawal na pag-ibig sa kapwa } \\
\text { lalaki }\end{array}$ & Inosentehan ng kabataan & $\begin{array}{l}\text { Ang naniniwala sa sabisabi ay walang } \\
\text { bait sa sarili }\end{array}$ \\
\hline Damdamin & Pag-ibig, pangarap & Pakikipaglaban, pangarap & Pangarap,pagkabahala at paghiling \\
\hline
\end{tabular}

\subsection{Pangnilalamang literari sa bawat akda}

Matutuklasan dito sa representasyon ng mga susmusunod na nilalaman ng akda tulad ng tauhan, tunggalian, banghay, tema, at damdamin na pumukaw sa interes ng bawat mambabasa na suriin ang lakang-akda ni Villafuerte at kikitaan ng mga pamatnubay sa gagawing pagsusuri. Binanggit ni Dinglasan (2001) sa kanyang aklat "Kritisismong pampantikan" ang kariktan ng isang akdang pampanitkan ay masusukat sa pamamagitanng pagsusuri sa kabuuan at nilalaman ng mga ito. Katangi-tangi ang mga lamang nagaganap at nagsisilbing daan ito sa pag-unawa sa mga babasahing nakalimbag.

Unang akda - Huling hiling, hinaing ni hermano huseng. Ang nagsiganap sa akda na gumagalaw sa mga pangyayari sa kuwento at may tauhan ding pinag-uusapan lamang ngunit wala sa pisikal na anyo. Ang tagpuan naman ay kaligiran ng kuwento na kung saan umiikot ang buhay ng mga tauhan dito nabubuo ang mga pangyayari. Ipinapakita rito na ang ipinaglaban ang bayan at personal na aspeto na kung saan ay hindi nakamit ang inaasam at muntik pang masali sa labanan. Higit na nagiging malalim ang pananaw sa buhay ng nagsiganap sa akda. Higit naging mga isyung pulitikal, personal at ekonomikal na danas ay mapusok at marahas. Hanggang ngayon ay nararanasan ng mga taong nasa mababang uri ang mga katulad na nakikipaglaban sa karapatang pantao at sa bayan. Ang miserableng tagaganap sa bawat akda na madamdaming inilarawan sa kuwentong ito:

$$
\begin{aligned}
& \text {...na anak-bukid at taga-anluwage sa bayan ang kanyang ama.. Bunso siya sa apat na } \\
& \text { magkakapatid... }
\end{aligned}
$$

Inilahad sa kasunod na elemento ay ang tagpuan na inilarawan sa akda ni Villafuerte ay nasa kahirapan sa bawat tagpuan. Sa pahayag na...

...sa nayong ito ay malayo sa sibilisadong kaalaman... Hindi nakakarating ang mga balita na galing sa karatig bayan at sa makabagong mundo sa Maynila.

Inilahahad ang mahahalagang pangyayari sa kuwento ayon sa pagkasunod-sunod ng mga ito, kagaya ng panimula, tunggalian, kasukdulan, kakalasan at katapusan. Kakitaan ang panloob na suliranin na nagpapakitang mahirap malutas. Kagaya ng lihim na pagtingin sa pangunahing tauhan na mahirap lutasin dahil hindi ito lantarang mailahad sa kababata. Ang pag-aasawa ng mga kapatid ni Hermano at sa hindi inaasahang sakit na umabot sa pagkamatay ng ama at sumunod sa isang taon ang ina ni Hermano. Ang napakariing dagok na halos kumawala sa matinong pag-iisip na ginawa ng ikalawang ina at minsan isang umaga ay pinaligo at pinabihis ng maayos ang pangunahing tauhan na si Ato at may lakad sila ng kaniyang ikalawang ina kung saan ay ipapasok

18 Consortia Academia Publishing (A partner of Network of Professional Researchers and Educators) 
siya nito ng trabaho.

Paunlad na Pangyayari. Hanggang sa nabalitaan niyang paalis na sa kanilang nayon at ito ay magtrabaho sa konstruksyon ng kauna-unahang mall na maipatatayo para sa mga Nueva Ecijano. Ito'y nangangahulugang sa lungsod siya maninirahan, at paminsan-minsan na lamang luluwas sa Tungkong-Bato.

Tunggalian. Tao sa kanilang sarili, ipinapakita ang panloob na suliranin ng dalawang tauhan na nagpapakita ng tunggaliang mahirap malutas. Ang lihim na pagtingin sa pangunahing tauhan na mahirap lutasin dahil hindi niya ito lantarang mailahad sa kababata. Naging suliranin din ang pag-aasawa ng mga kapatid ni Hermano at siya na lang ang nanatiling binata. Sa hindi inaasahang sakit na umabot sa pagkamatay ng ama at sumunod sa isang taon ang ina ni Hermano.

Kasukdulan. Ang pag-anib sa kilusan. Karanasang hindi niya malimutan nang siya ay ginahasa. Pagtatapat niya sa kanyang Tatang at Inang sa tunay na pagkatao at iniingatang lihim. Sumunod ang pagtakwil ng mga magulang niya na naging dahilan upang sumamang mamundok kay Hermano Huseng. Sa kasukdulan ang pinakatuktok sa kuwento kung saan ay mababasa sa akda na ang pag-anib sa kilusan. Karanasang hindi niya malimutan nang siya ay ginahasa. Pagtatapat niya sa kanyang Tatang at Inang sa tunay na pagkatao at iniingatang lihim. Sumunod ang pagtakwil ng mga magulang niya na naging dahilan upang sumamang mamundok kay Hermano Huseng.

Kakalasan. Napagsino ang kanyang sarili - ang noo'y kayraming pangamba at takot na ayaw kumawala sa kanyang dibdib, pagkamakasarili, ang maling paglingon sa hinaharap, ang napakataas na pagpapahalaga sa edukasyon at hindi sa damdamin ng nakararami hanggang sa Katapusan na nawalang-saysay ang lahat ng pagsisikap at pag-asam.. Nagising siya sa katotohanan na hindi pa maaring mangyari ang lahat. Kaya siya bumaba sa siyudad at nilisan ang bundok, iniwan ang mga sakit at maliligayang alaala.

Tema. "Sa ngalan nang pag-iibig, susundan kita masunod ka lamang" na nagsasaad patungkol sa pag-ibig, pag-asam, pagkamit at paglaban sa ano mang kahihinatnan maging mapanganib o ito man ay maging sanhi ng kamatayan. Kahit sino ay pwedeng makamtan ang pag-ibig na inaasam, wala itong pinipiling edad, kasarian, lugar o lahi.

Masidhing damdamin ang umiinog sa daloy ng kwento. Puno ng pag-asa at pag-aalsa para sa katotohanan maging sa pag-ibig at patungkol sa bayan.

Makikita na ang kuwento ay unti-unting pagbaba ng kuwento mula sa napagsino ang kanyang sarili - ang noo'y kayraming pangamba at takot na ayaw kumawala sa kanyang dibdib, pagkamakasarili, ang maling paglingon sa hinaharap, ang napakataas na pagpapahalaga sa edukasyon at hindi sa damdamin ng nakararami hanggang sa Katapusan na nawalang-saysay ang lahat ng pagsisikap at pag-asam.. Nagising siya sa katotohanan na hindi pa maaaring mangyari ang lahat. Kaya siya bumaba sa siyudad at nilisan ang bundok, iniwan ang mga sakit at maliligayang alaala.

Ikalawang akda-Si ato sa pagtakas sa kawalang-malay. Ang tauhan ay si Ato siya ang pangunahing tauhan ng kwento na lalaki subalit maadalas niyang marinig na anak siya sa unang lalaki ng kanyang ina. Matutunghayan ang mga ito sa mga sumusunod na pahayag...

"Pero iyong batang lalaking nakita mo kanina, si Ato ay anak ng misis ko sa kanyang unang boyfriend... May tagong landi ang asawa ko...”

Nagnanais siyang makatapos na pag-aaral para maka-tuntong sa mga magaganda at matataas na gusali. Gustong kumita ng malaking pera para makabili ng bagong damit. Puno ng pangarap na kahit katiting lang ay maabot ito. Kahit simpleng pangarap lang ay matutupad basta’t ito ay may kaakibat na pagtitiyaga.

Masaksihan na sa inosenteng pag-iisip ay kaya niya ang trabaho at walang pag-reklamo sa kanyang ama. 
Binubuhay ang pamilya sa murang edad kung saan napasabak sa peligrong trabaho sa kahabaan ng kalsada. Ang tagpuan ay Isang iskwater na lugar ang pinangyarihan ng kuwento. Nabanggit sa isa sa mga talata na... "Kaya't nang paalisin sila sa Tatalon, kasama ng ilan pang mga iskuwater ay nagpatulong ang kanyang ama sa kanyang mga kaibigan na makapagpatayo ng isang barung-barong sa may Coastal Road". Ang lugar na ito ay napakalapit lang sa makabagong panahon. Kung saan na lahat ng nagtataasang gusali ay makikita at magandang tanawin pati mga magagarang kotse ay halos hindi na mabilang sa kadadaan.

Pasimula. Nagsimula ang kwento sa pagnanais na matupad ang kanyang mithiin na makaahon sa hirap. Dahilan rin na ang kanyang ama ay hindi na sila kayang buhayin. Kaya kahit sa anong trabaho ay gusto niyang pasukin sa kamusmusang pag-iisip ay hindi niya inakala na mapanganib na pala ito. Hanggang sa paunlad na Pangyayari ay ipinapakita na...

...Ibig kumita nang malaki si Ato. Ibig niyang makaipon...

...Ibig niyang makipon upang makabili ng bagong t-shirt at maong...

Isa dahilan ito na tatlong luma't nangingitim na t-shirt ang pinagpapalit-palit siyang isuot. Isang masikip na luma't kupasing maong at isang gapok nang short ang halinhinan niyang isinusuot. Dahil sa nainggit siya sa kanyang kaibigan na si Jhun ay gusto rin nyang pasukin ang trabahong ginagawa nito at kahit ito man ay mapanganib.

Tunggalian. Ang napakariing dagok na halos kumawala sa kanyang matinong pag-iisip na ginawa ng kanyang ikalawang ina at minsan isang umaga ay pinaligo at pinabihis ng maayos ang pangunahing tauhan. Ipinahuhubad sa kanya ang kanyang pantalon. Umiling siya. Muling sumenyas ang Hapon na waring nagsusumamo. Naisip niya, baka bahagi iyon ng kanyang trabaho. Nakatitig siya sa telebisyon habang hinuhubad niya ang kanyang pantalon.

Kasukdulan. Pagkahiga niya ay hinatak ng Hapon ang kanyang panloob na kasuotan hanggang sa bigla na lang nanghihina at nawalan ng malay. Pagkagising niya ay masakit na masakit ang kanyang buong katawan. Napaire sa sakit nang di-sinasadya'y napatagilid siya't nakapa ang puwit at kung anong bagay na madulas. Nang tiningnan niya ito ay sariwang dugo. Pinagmumura niya sa tindi ng galit ang dayuhan at pinagpupunit ang mga larawang hubad hanggang sa tatlong sunud-sunod na suntok ang tumama sa mukha niya at pansamantalang nakatulugan ito.

Kakalasan. Nang makita ni Ato ang kanyang kapatid na si May ay hindi na niya hinayaan pang mangyari ito sa kapatid buong diing hinawakan ang tinidor. Pagkabukas na pagkabukas ng pinto ay hinanap ng kanyang nanlilisik na mga mata ang Hapon. Inundayan niya ito ng saksak sa dibdib, braso, leeg, batok, noo, mukha at sikmura. At nang mapahiga ang Hapon ay buong lakas na inundayan niyang muli ng saksak sa dibdib. Sunud-sunod at paulit-ulit.

Ang tema "Ang kamusmusan ng kabataan ay hindi dapat lalapastangin ng nino man". Ang tema ng kuwento ay nagsasaad sa mambabasa na ang ka-inosentehan ng kabataan ay dapat mabigyan ng kaukulang pag-aalaga sa tamang daan at pagnanasang makamit ang ninais na karunungan. Sa mag-aaral na ay dapat may sapat na kaalaman sa tamang gawi, karapatan at sa mga guro naman ay maging tulay tungo sa pagbabago ng kaisipang puno ng pagtatanong at pag-asam ng karunungan. Ang Tema ay makabuluhang patungkol sa buhay na maaaring makalantad ng pahayag o pahiwatig. Binanggit ni Cuizon (2014) na tema ang nabubuo mula sa pagsusuri na nagsasaad ng katotohanang panlahat.

Ipahayag na ang damdamin ay puno ng pakikipaglaban at pangarap. Ito ay naglalarawan ng pighati, pagkamuhi, pag-alipusta, dahas, at paggamit ng mga inosente sa illegal na kalakaran. Makakamit lamang ang mga ito kapag ang tao ay marunong makipaglaban sa katarungan.

Ikatlong akda - Ang huling itineraryo sa pulu-pulong utak ni propesor balisungsong. Ang gumanap ay si 
Pagtuklas sa mga piling maikling akda ni P. V. Villafuerte sa realismong pananaw

Propesor Balisungsong isang propesor sa unibersidad na nagtuturo ng Philosophy. Prestihiyoso at may malaking karangalan. Nakasaad sa talata na...

...Saan ka ngayon?...Di ba't philosophy ang itinuturo mo...? Malinis nga ang damit mo. Maporma. Mabango. Iginagalang. Hinahangaan. Kinatatakutan. Ngunit mababa ang pasahod. Alipin ka pa ng oras. $\mathrm{Ng}$ sistema. Ng intriga. At ng lahat-lahat na...”

Istriktong guro sa kanyang mga estudyante, matakutin sa kanyang biyenan na isang titig lang ay malulusaw na. Umaasa na mapataas ang sweldo sa kanilang madalas na pagmiting. Nabanggit niya sa pangungusap na...

... “may meeting sa Conference Room mamayang alas-onse ng tanghali...

Tanging hanggad lang ang mapataas ang sahod nang sa ganun ay makapag-uwi ng malaki-laking pera sa kanyang misis. Gusto lang ang mapasaya ang pamilya at mabigyan ng magandang buhay. Hangad ito na marinig ang nais ngunit hanggang sa dila lamang ng administrador ng eskwelahang pinapasukan ng tauhan sa akda. Pagtuklas ng gawain ng isang guro na may malasakit sa kanyang kapwa ay walang hinihinging kapalit.

Sa eskwelaha ang tagpuan ito ay isang unibersidad na pinagtatrabahoang pampublikong paaralan. Hindi man tahasang binanggit ang tiyak na pangalan ng lugar, sa kuwento ay ipinahiwatig ng ilang pahayag na naganap sa sumusunod na pahayag na...

... “Ewan ko ba kung bakit gabing-gabi’y nariyan ka pa sa Men's Faculty Room. Tapos na kanina pa ang evening class mo...

Nagtrabaho sa malaking unibersidad at dahil sa nabanggit ang faculty men's room, klase, mga estudyante at main gate ng unibersidad. Ipinapahiwatig din nito na malakilaki ang pasahaod kasi sinabing ito ay unibersidad at koliheyo ang tinuturuan ng tauhan sa akda. Pero ito ay kabaliktaran kung saan ipinagwalang bahala ito ng administrasyon. Malaking pagkakahawig ito sa lipunang Pilipino kung saan ang mga mamamayan ay kakarapot lamang ang maiuuwi na pera sa bahay na halos hindi na makakain para lamang may maitabing pera sa pamilya.

Panimula. Nagsimula ang kwento sa panahong na pakaroon ng pagbaba ng peso, kung saan maaepektuhan ang pasahod ng pangunahing tauhan na pinag-ugatan ng suliraning kanyang kinakaharap sa kasalukuyan. Paunlad na Pangyayari. Naging Department Head na sa eskwelahang pinagtuturuan noon, lumipat sa unibersidad na pinagtapusan. Kesyo mas mataas ang sweldo at mas may pangalan. Simula ng lumipat sa kanyang pinagtrabahuan ay nagkanda hirap sa buhay ang tauhan ng kuwento. Kulang lamang ang sahod sa pantustos ng pangangailangan ng pamilya. Kaya niya sinabak ang pagkaroon ng hiling sa mataas sa unibersidad na taasan ang sweldo. Hanggang sa siya ay ginugutom na ng karangalan at prestihiyo.

Kasukdulan. Nang pagkagising niya sa umaga ay dapat sana may langgonisa't tosino, sausage o pritong pata na nakahain sa mesa. Pero dahil sa peso devaluation ay isang pritong gigi (galunggong) at pulang kamatis na lamang. Napahiya siya sa asawa at sarili niya dahil niwala siyang maiabot na pera. Natatawang naiinis siya dahil napakatapang niya sa klase ngunit titig lang ng biyenan niya ay di magawang umalis dahil na rin sa kapos sa sweldo ay di kaya ang pagkuha ng panghulog sa subdibisyon.

Kakalasan. Ang nagrali na kabataan tungkol sa pagtaas ng tuition fee. Lahat ng nakita niya sa paaralan ay walang kwenta sa kanya dahil gulong-gulo ang isip niya. Sa pagkakaroon ng miting ay atat na atat siya mapagpasyahan ito ngunit ganun pa rin puno na naman ang utak niya sa kaiisip kung kalian dahil sa mayroong pagbabasbas ng bagong gusali sa loob ng unibersidad.

Katapusan. Kahit gabing-gabi na ay hindi pa rin siya umuwi. Pumasok sa Men's faculty room ng unibersidad at sa bagong gusali siya nagtuloy. Kumuha ng mga gamit pangpintura para sulatan ang makinis na makinis na dingding. Sa pagkakaalam niya na ang dapat managot sa bandalismo sa dingding ay ang mga estudyanteng kasama niya nang namasyal sila doon. Ngunit, nakalimutan niyang mai-lock ang pinto ng bigla na 
Mandado, J. O.

lang nabunggo niya ang lata na lumikha ng ingay, napansin ng taong nagbantay sa gusali at bumukas ang pinto at bago siya nakapagsalita sunud-sunod na putok ang kanyang narinig. Napatay siya at iyon ang huli niyang plano sa buhay pati mga hiling niya ay kasama sa bendisyon sa bagong gusali ng unibersidad kinabukasan.

Ang Tema ay “Ang naniniwala sa sabisabi ay walang bait sa sarili”. Ang tema ng kwento ay nagsasaad sa mambabasa na puno ito ng mga agam-agam, pagkabagot, pagkamit ng katarungan, kalayaang magpahayag, at pagpaplano. Kapayapaan, katotohanan at katarungan ang dapat mamayani sa sanlibutan para makamit ang lahat nang ito.

Namayaning damdamin ay puno ng pagkabahala at paghiling. Ito ay nangangahulgang paghiling, pagkamit sa katarungan, pag-iisip na naglalarawan sa isang tao. Ang layunin ng magsalaysay ng mahalagang pangyayari tungkol sa pangunahing tauhan ukol sa maikling kuwento ay mas kakaunti ang tauhan ng akdang ito ay maikling panahon ang sinasakop kung ikukumpara sa nobela. Layunin din nitong magbigay-libang at sigla sa mambabasa (San Juan et al., 2005). Inilalarawan ang pangyayaring pangkaugalian ng mga karakter na nagsiganap upang mabigyang kabuuan ang pag-uunawa.

Ang bawat ipinapakita sa panganuhing tauhan sa kuwento ay may iba't ibang pagganap. Ang bawat katha ay may pagpapakilala sa mga tauhan kung saan ay malinaw ang pahayag ng pangyayari, sa pananaw ng tauhan na ginamit, sa pananalita ay may halong banyaga at makabago sa kamalayan. Kumikilos sa pangyayaring angkop sa suliranin ng bawat kwento. Ito ay masining sa iba't ibang paraan na nabigyang-buhay sa panlabas na kanyuan, ugali, damdamin at mithiin. Mayroon ding kaparehas ang gamit ng manunulat sa kanyang kuwento na nagpapahayag tulad ng salita na may balbal, banyaga at talinghaga ang mga ito. Sa ikalawang at ikatlong kuwento ay ang lugar ng pinangyarihan ay hindi tiyak, puno ng mapanghusga sa kapwa ang lugar, sa mga salitang gamit ay pabalbal, at ito ay may makasariling pagnanais na makamit ang inaasam ng isang tao sa kanyang lipunan.

\subsection{Pagsusuri ayon sa ugnayang panlipunan at kaisipan}

Bawat tao ay may kanya-kanyang pagganap. Maging sa loob ng tahanan o sa lipunang puno ng halaga sa buhay. Binanggit ni Reyes (2006) sa aklat ni Yu (2006) na ang implikasyon ng kritkal na nagmula sa sangkapang akda, ang lipunan, ang manunulat at mambabasa-karaniwang higit na pinahahalagahan ang ugnayan ng akda at ng buhay ng mambabasa. Inilalarawan sa Talahanayan 2 ang pangyayaring ugnayan ng mga karakter na nagsiganap upang mabigyang kabuuan ang pag-uunawa.

\section{Talhanayan 2}

Ugnayang panlipunan at pangkaisipan

\begin{tabular}{lll}
\hline \multicolumn{1}{c}{ Mga Kuwento } & \multicolumn{1}{c}{ Ugnayang Panlipunan } & \multicolumn{1}{c}{ Ugnayang Pangkaisipan } \\
\hline $\begin{array}{l}\text { Ang Huling Itineraryo ng Pulu-Pulong Utak } \\
\text { ni Propesor Balisunsong }\end{array}$ & $\begin{array}{l}\text { Mapagmataas } \\
\text { Mapanghusga } \\
\text { Inabuso }\end{array}$ & Kawalan ng katarungan \\
Si Ato sa Sangmagdamagang sapwa \\
Kawalang-malay & $\begin{array}{l}\text { Inosente sa mundo } \\
\text { Puno ng pangarap }\end{array}$ & Nawalan ng \\
Huling Hiling, Hinaing at Halinghing ni & Inabuso & tiwala sa Diyos \\
Hermano Huseng & Inabuso & Panikibaka sa murang edad \\
& Aktibista & Marupok kapwa \\
\hline
\end{tabular}

Sa inilahad na mga akda ay may pag-aabuso, mga bagay sa mundo na realidad na sumisimbolo sa pangyayari sa isang lipunan at naghahanap ng katarungan sa lipunang ginagalawan. Sa teorya ni Scott (1974) ang ugnayang panlipunan ay nakapagdaragdag ng lakas na may bahaging ginagampanan sa pakikipag-ugnayan ng lipunan. Mula rito ay maraming napupulot na pangyayari, sitwasyon at maaaring gamiting paksa. Binanggit ni Pineda (1999) upang magkaroon ng makatotohanang direksyon ang bungang-isip ng manunulat ay kailangang makilalang tunay ang lipunang iyon.

Sa unang kuwento ay ang isang mapagmataas at maprinsipyong guro. Ilang taon ding pinag-aralan na 
maging propesyunal ang isang tao at naipagmalaki ito kapag mataas ang naabot. Ganito rin ang kuwento ni Propesor Balisungsong na kahit nahihirapan na ay patuloy paring mapagmataas sa mga kaibigan. Nahahangad ng magandang pasahod pero hindi pala nang lumipat sa isang unibersidad kung saan ito isang pampublikong paaralan ay lalo pang naghirap. Hindi na sapat ang sweldo at ang pamilya ay naapektuhan pa sa pagtaas ng mga bilihin sa merkado. Kabliktaran sa panlabas na anyo kung saan kahit sinong makakita sa kanya ay bilib na bilib dahil maporma pa rin sa kasuotan kahit kumakalam ang tiyan sa gutom.

Ang kaisipang binibigyang-diin ng maikling kuwento ay una, nakatuon sa pagyakap sa pangarap at hangarin ng pamumuhay na idinidikta. Dapat makuntento sa mga hangarin sa buhay at maging sa lipunan ay sumunod sa batas na ipinatupad para sa kaayusan ng lahat. Ikalawa, huwag gumamit ng dahas na kung saan ay babalik sa taong gumawa na naging mitya ng buhay.

Sa ikalwang kuwento ay ang paggamit sa mga inosenteng kabataan na matutunghayan sa kwento, na ginagamit ang mga kabataan sa murang edad sa pagtatrabaho, tulad ng pamamalimos, pagtitinda ng laman, nasa kahabaan ng kalsada, o kahit sa pagbibenta ng iligal na gawain ay masaksihan ang mga ito na labis na nagbibigay katanungan sa kanilang murang isipan. Sa kamusmosan ay dapat nasa iskwelahan ang kabataang ito para matuto ng mga leksyon at magamit kalaunan. Ngunit baliktad ito sa kuwento, mismo ang ama ang siyang nag-udyok na magtrabaho para may makain at mabuhay sila. Ang kawalang pinag-aralan o kakulangan sa pag-aaral ay minsang matatawag na ka-inosentehan. Lalo na sa murang edad ay puno ng pagtatanong sa mga nasaksihan at nakikita.

Ang kuwentong ito, kay dami ng mga tanong na nais niyang masagot tulad ng paghihiwalay ng magulang niya at sa kawalang kaalam-alam naibenta na siya sa dayuhang Intsik. Nang marinig ang boses ng kanyang bunsong kapatid na babae ay ipinaglaban niya ito hanggang sa nabawian ng buhay ang dayuhan. Ang ikatlong kuwento ay nagsasaad ng panlipunang ugnayan sa pag-ibig na bawal. Lubhang nasiyahan ang tao kapag ang isang iniibig ay parehas ang inyong naramdaman. Ang pag-ibig na nagbibigay sigla, saya, lungkot o minsa'y humahantong sa wala. Umaasa na sana’y mapansin sa kanyang kababata na si Hermano. Ginawa niyang lahat para lang mapalapit sa tao ngunit kabaliktaran ang nangyari dahil may minamahal na ito. Nasaktan at nabigo siya sa kanyang pakikipagsapalaran, ang kanyang ipinaglaban na lihim na pag-ibig. Kahit sa paglalaban niya sa katotohanan ni Hermano Huseng sa bayan ay walang magandang nangyari dahil natalo sila sa pakikipaglaban sa mga alagad ng gobyerno. Ang pagka-ubos ng kabuhayan. Sa kuwento isinalaysay na ang kanilang nayon ay...

...Nangabitak ang dating matabang lupa. Nangarilang ang buong palayan.

...Nangamatay ang maraming hayop at halaman. Nangagkasakit ang maraming bata...

...Nangagsara ang ilang negosyong pang-agrikultura sa Gapan at San Isidro.

Inilahad sa kuwento na ang kanilang nayon ay sinalanta ng tagtuyot at walang magawa ang mga taga-bukid kung saan ay binawi ng ilang negosyante't imbestor ang pakikipagkalakalan sa kanilang bayan. Dahilan sa nagutom ang lahat ng taga-Tungkong Bato at hindi man lang pinansin ng pamahalaan na may kumakalam na sikmura ng mga tao. Ang pag-aalsa laban sa gobyerno. Ang kwento ng dalawang pangunahing tauhan ay labis nilang ipinaglaban ang kanilang karapatan, katotohanan, at kalayaan sa bayan. Inilalahad sa sumusunod na salita...

Sama-samang pagsigaw

Sa langit nakatunghay,

Sama-samang pagkilos

Sa lupa nakalaan...

Naranasan ng pangunahing tauhan ang walang hustisya para sa mga mahihirap at naaapi sa kanilang bayan. 
Mandado, J. O.

Paglalaban ng sariling karapatan at gayon din mga taong inaapi at inalipusta ng mga taong may mataas na katungkulan sa mamayan na siya ring simura sa dignidad ng sangkatauhan. Nahirapan ng lubos ang pangunahing tauhan sa kanyang naramdamang lihim na pag-ibig sa kapawa niya.

Ang kaisipang binibigyang-diin ng maikling kwento ay una, nakatuon sa pagyakap sa pangarap at hangarin ng pamumuhay na idinidikta sa atin ng lipunan. Marapat na ang magtakda ng pamantayang tunay na magtataas sa mga dignidad ay tayo. Ikalawa, sa kabila ng mga suliranin hindi dapat mawalan ng pag-asa bagkus maging positibo at simulang tahakin ang naghihintay na bukas na malutas ng paunti-unti ang mga suliraning kinakaharap at dadating. Tiyaking naayon ang mga ginagawa sa pamayanan na hindi ito nakakasira ng sino man. Manatiling malakas at magtiwala sa sarili na makamtan ang nais at pangarap para sa kinabukasan.

\subsection{Pagsusuri ayon sa ipinapakitang realismong pananaw}

Ang realismo ay katotohanan batay sa makatotohanang paglalahad at paglalarawan ng mga bagay, tao sa lipunan, nag-uugnay ng kapwa, pagbabago at pag-uunlad. Matutunghayan sa mga katha ni Villafuerte na isa sa pangkat ng pagsusuri ng realismo ay ang kritikal na realismo- ay mga gawain ng isang lipunang burgis upang maipamalas ang mga aspetong may kapangitan at panlulupig nito (Bernales et al., 2013).

\section{Talahanayan 3}

Pagpapakitang Realismong Pananaw

\begin{tabular}{|c|c|c|}
\hline Mga Kuwento & Kapangitan & Panlulupig \\
\hline Ang Huling Itineraryo ng Pulu-Pulong & Maprinsipyong guro & Pang-abuso \\
\hline Utak ni Propesor Balisunsong & $\begin{array}{l}\text { Pag-aalsa ng mga guro } \\
\text { Mapanghusga }\end{array}$ & Human Trafficking \\
\hline Kawalang-malay & $\begin{array}{l}\text { Walang pinag-aralan } \\
\text { Nakapatay }\end{array}$ & $\begin{array}{l}\text { Kulang ang sahod } \\
\text { Napatay }\end{array}$ \\
\hline Huling Hiling, Hinaing at Halinghing ni & Lihim na Pag-ibig sa kauri & Naapi at inalipustang bayan \\
\hline
\end{tabular}

Makikita sa Talahanayan 3 na lumulutang sa kanyang tatlong kathang sinulat na naglalahad na mayroong kapangitan at panlulupig sa kanyang katha- tulad ng unang kuwento. Sa kantang Baligtad na ang Mundo dinalirot niya ang sensitibong isyu ng kawalang-hustiya sa lipunan at ang tila pagpapalaganap ng kultura ng kawalang pakialam ng gobyerno ukol sa nagaganap na kasamaang ito. Bagamat may katagalan ng naisulat ang katha ay nanatiling buhay ang paksa nito na makaugnay ang sinumang babasa.

Ang kapangitan sa loob at labas ng lipunan ay masasalamin ninuman lalo na kung ang gumaganap ay ang mismong nakaranas nito, tulad sa mga nangayari sa kuwento na lahat ay puno ito ng kamalasan at kapangitan. Isang halimbawa ang akda ni Villafuerte na taluntunin ang mga abusado sa kanilang kapwa, mapagmataas, walang takot sa batas at may panlulupig. Minulat ng may akada ang mga mambabasa na bigyang salamin ang katohanan upang makita mismo sa kabila kung ano ang nangyayri sa bulok na lipunan. Subalit hindi ito ibig sabihin na dapat mawalan ng pag-asa at tiisin ang pang-aapi. Tunggkulin ng bawat isa na magsagawa ng pagbabago tungo sa ikabubuti ng lipunang ginagalwan.

Binigyang-tibay ang pahayag na ito ni Sicat (2016) at Melendrez-Cruz (1994) na kailangang suriin ang malikhaing pagkatha sapagkat ang mahusay na panitikan ay kinakailangang naglalarawan sa mga realidad ng lipunan at nagbibigay ng matalas na pagsusuri dito. Mula sa pahayag na ito, masasabing masining at panlipunang kalikasan ang panitikan ang kailangang pagtuunan ng mag-aaral na makilala at mapag-isa ang pambansang identidad. Ang konseptong ito ay nagpapatotoo sa sinabi ni San Juan et al. (2005) na magsalaysay ng mahalagang pangyayari tungkol sa pangunahing tauhan.

\section{Natuklasan}

Batay sa ginagawang pagsusuri natuklasan sa pag-aaral na ito na ang ginamit ng may-akda sa tatlong 
kuwento ay may pagkakatulad at pagkakaiba. Ang akdang Si Ato sa Sangmagdamagang Pagtakas sa Kawalang-malay at Huling Hiling, Hinaing at Halinhing ni Hermano Huseng, at Ang Huling Itineraryo ng Pulu-Pulong Utak ni Propesor Balisunsong ay parehong kuwento ng pangunahing tauhan. Magkaiba ang gamit nila ng wika, may pormal at di-pormal ang salita. May paggamit ng likhang salita at salitang pa-Ingles. Matalinghaga at matayutay ang mga gamit na salita sa isang kuwento. Tugmang-tugma sa lugar na pinangyarian ang bawat salitang gamit sa akda, inaayon ito sa kapanahunan. Ang tatlong kwento ay nasa karaniwang pagsalaysay sa pangyayari, paglalahad at paglalarawan niya.

Magkaiba ang tema ng tatlong akda, ang una ay sa ka-inosentehan ng bata na dumaan sa pagsubok at pagtakas sa mapapait na pangyayari, ang pangalawa naman ay nakatawag pansin ang pagkaroong matunggaling paglahad ng tauhan, ito ay pagkabahala at pagkamit ng inaasam ng isang propesor sa unibersidad at pangatlo naman ay ang pagyurak sa dangal at madamdaming paghihimagsik laban sa kapwa lalaki. Parehong makabuluhan sa kasalukuyang panahon ang mga temang ito.

$>$ Ang mga elementong gamit ng bawat maikling katha ay ang tauhan, tagpuan, banghay, tema at damdaming pantaong nakapaloob sa mga katha. Sinasalamin ang mga akdang pampanitikan sa bawat yugto ng buhay at sa lipunan na pumapalibot sa tao.

$>\quad$ Ang tatlong akda ay may pag-aabuso sa tao at sa mga bagay sa mundo na sumisimbolo sa pangyayari sa isang lipunang naghahanap ng katarungan.

$>\quad$ Lumulutang sa kanyang tatlong akdang sinulat na naglalahad na mayroong kapangitan at panlulupig sa kanyang kuwento na sumasalamin pangyayari sa totong buhay ng tao.

\section{Konklusyon}

Hatid ng akda sa lipunan at mambabasa na ang mga maikling akdang nasuri ay mahusay ang paglalarawan sa pagsasaad ng mithiing makamit ang katarungan at pagpapahalaga sa kinabibilangang lipunan.

\subsection{Rekomendasyon}

Batay sa mga resulta ng pagsusuri, nabuo ang sumusunod na rekomendasyon:

$>\quad$ Magamit ang maikling kuwento ni P. V. Villafuerte hindi lang sa ibang kursong kolehiyo kundi higit sa kursong pampagtuturo sa mga maikling kwentong na malinang ang kahalagahang pangkatauhan at mailapat ang mga pangyayari sa mga maikling kwento sa tunay na buhay.

$>$ Magkaroon ng mga seminar at worksyap para sa mga guro na magamit ang pagsusuring ginawa sa kanilang pagtuturo sa mga mag-aaral sa Panitikang Pilipino.

> Magkaroon ng pananaliksik o pagsusuri sa kolehiyo ukol sa paggamit ng mga akdang pampanitikan sa pagtuturo ng wika at panitikan.

\section{Mga sanggunian}

Arrogante, J. A., et al. (2004). Filipino literature [Panitikang Filipino] (New ed.). National Bookstore.

Bayang, E. E., \& Go-Aco, E. G. (2020). Observations of Filipino ambassadorial cultures from lessons in Asian literature in Filipino by K12 - Grade 9 module [Mga obserbasyon ng mga kulturang ambansang Pilipino mula sa mga aralin sa panitikang asyano sa Filipino ng K12 - Grade 9 module] (Vol. IX). Manila. https://doi.org/10.24214/jecet.C.9.2.25569

Belvez, P. M., et al. (2006). Race literature [Panitikan ng lahi] (College level). Rex Bookstore.

Bernales, R. A., et al. (2013). Filipino 2 in college: Reading and writing towards research [Filipino 2 sa kolehiyo: Pagbasa at pagsulat tungo sa pananaliksik]. Mutya Publishing. 
Mandado, J. O.

Cuizon, R. I. (2014). Meta analysis in the analysis of short stories in theses and dissertations [Meta analisis sa pagsusuri ng maiikling kwento sa mga tesis at disertasyon] (Vol. II). Zamboanga del sur.

Dinglasan, R. D., et al. (2005). Literary critique: Selected stories by Pineda [Kritisismong pampanitikan: Mga piling kwento ni Pineda]. Rex Bookstore.

Fraenkel, J. R., \& McGraw, N. (2003). How to design and evaluate research in education. Waller Hill Higher Education.

Panganiban, J. V., et al. (1987). Philippine literature [Panitikan ng Pilipinas] (New ed.). Bede's Publishing House.

Salazar, L. A. (1995). Filipino literature [Panitikang Filipino] ( $3^{\text {rd }}$ ed.). Katha Publication Company.

San Juan, G. P., et al. (2005). Literary analysis [Panunuring pampanitikan]. Booklore Publishing Corporation.

Santiago, E. P., et al. (1989). Filipino literature: History and college development [Panitikang Filipino:

Kasaysayan at pag-unlad pangkolehiyo]. National Bookstore.

Sauco, C. P., et al. (1989). Filipino literature: History and development. [Panitikang Filipino: Kasaysayan at pag-unlad]. Navotas Press.

Sicat-de Laza, C., \& Batnag, A. (2016). Reading and analyzing various texts for research [Pagbasa at pagsusuri ng iba't ibang teksto tungo sa pananaliksik] ( $1^{\text {st }}$ ed.). Rex Bookstore.

Stroud, S. R. (2011). John Dewey and the question of artful criticism: Philosophy and rhetoric (Vol. 44). Penn State University Press. https://doi.org/10.5325/philrhet.44.1.0027

Tablan, F. (2011). Socialist ideas in the works of Amado V. Hernandez [Kaisipang sosyalismo sa mga akda ni Amado V. Hernandez] (Vol.5, Issue 1). Department of Philosophy, Bellevue College, USA. https://doi.org/10.25138/5.1.a.2

Villafuerte, P. V. (2000). Literary criticism: Theory and practice [Panunuring pampanitikan: Teorya at pagsasanay]. Mutya Publishing House.

Yu, R. T. (2006). Kilatis: Philippine literary criticism [Kilatis: Panunuring pampanitikan ng Pilipinas]. The University of the Philippines Press. 